\title{
Survey of Medicinally Important Weeds from Western Region of Kopargaon, District- Ahmednagar (M.S.), India
}

\author{
R. R. Sanap \\ P.G. Department of Botany, R .B. N.B. College, Shrirampur \\ Dist. Ahmednagar (M.S.), India
}

\begin{abstract}
The survey of medicinally important weeds was carried out from Western Region of Kopargaon during the year 2018 to 2019 . The present studies revealed that, near about 58 species belonging to 35 families were recorded from this region. Plant identification was carried out by various floras and manuals. Most of these weed plants recorded are used by people to cure various diseases and used as crude drugs. The various parts of plants such as roots, stems, leaves, flowers, fruits, seeds, rhizomes, tubers, lattices or sometimes entire plant is used as medicines to cure various diseases. These therapeutically important plants constitute various active principles which cause the physiological effect on the body and cure the diseases.
\end{abstract}

Keywords:- Survey. Medicine, Weeds, Flora, Kopargaon.

\section{INTRODUCTION}

From very ancient period of time plants are playing very important role in human welfare. All the three basic needs like food, shelter and clothing are fulfilled by plants and its products. Besides these, medicine is important need of human being. From very ancient period of time, plants are used to cure various diseases.

Weeds are unwanted plants growing in the field. Weeds cause the serious problem to the farmers. They absorb the nutrients from the field, growing vigorously and ultimately affect the crop production. But some weeds are used as crude drugs by many people. Folklore medicinal plants constitute a group of therapeutically important plants which are of great value for domestic use and also for export. Plant based drugs are being increasingly preferred in medical science. The use of various parts of plants has very specific capacity which causes the physiological effect on the body and cure the diseases. India have rich heritage of folklore medicinal plants.

Kopargaon is situated at Northern region of Ahmednagar district. It is an irrigated area and rich in biodiversity of medicinal and aromatic plants. Survey of medicinally important weeds was carried out from the Kolpewadi to Wadgaon village which is the boundary of Nashik district and is western part of Kopargaon taluka. Near about $15 \mathrm{~km}$ area is covered for the study purpose. The vegetation of the study area was rich in the herbaceous flora as it is irrigated throughout the year. Survey of medicinally important plants and its medicinal values was studied by many workers in India like Auti, 1911; Mohaptra and Behera, 2011; Rao et al, 2011; Behera and Sen, 2007; Rajaram et al, 2014; Basu and Ramsankar ( 2007). etc. But this area was untouched and so it was undertaken for the investigation

\section{MATERIALS AND METHODS}

Monthly collection of plants was carried out for the year 2018-19. During present studies, the medicinally important weeds from the selected area of Western Region of Kopargaon taluka were collected. The identification was done with the help of relevant floras, manuals and relevant literatures (Cooke, 1903; Sutaria,1962; Pradhan and Singh,1999; Kirtikar and Basu,1984; Deokar,1998; Kokate et al, 2016). Enumeration of plants is done by using botanical names, family names, plant parts used and curing diseases. Plants are arranged according to alphabetical manner.

\section{RESULTS AND DISCUSSION}

During present studies, extensive survey of medicinally important weed plants from the study area was carried out, plants are identified with relevant floras and enumerated in tabular form (Table.1).

Many workers have studied the medicinally important plants from various regions of the country. Auti (2011) studied the medicinal flora of Angiosperms from Jeur ( Bayajabai), District Ahmednagar while, extensive survey of folklore values of weeds grown in wastelands of Vedharanyam and Kodiakarai, Nagapattinam district of Tamil Nadu was made by Basu and Ramsankar ( 2007). Mohaptra and Behera (2011) studied the medicinal plants along the water stream of Pradhanpat water fall of Deogarh forest range in Deogarh district (Orissa). Behera and Sen (2007) investigated the traditional use of some plants against gynaecological disorders by the tribals of Ramkhol village forest of Barapahad hill range in Bargarh District (Orissa) while, Rao et al, (2011) have pointed out the indigenous phytotherapy for Gastro-Intestinal Disorders among tribals of Dhenkanal district. Orissa. Rajaram et al (2014) has studied herbal remedies for common ailments prevailing in rural areas of Palladam and Perumanallum, Tiruppur district, Tamil Nadu. Tripathi et al, (2007) studied the medicinal plants of Northern Hill Region of Chhattisgarh and their uses. 
Enumeration of medicinally important weed plants encountered during investigation period:

\begin{tabular}{|c|c|c|c|c|}
\hline $\begin{array}{l}\text { Sr. } \\
\text { No. }\end{array}$ & Botanical Names & Family & Plant Part Used & Medicinal Use \\
\hline 1 & Abutilon indicum Sweet & Malvaceae & Seeds, leaves & Cough, piles, tuberculosis \\
\hline 2 & Achyranthus aspera Linn & Amaranthaceae & Entire plant & $\begin{array}{l}\text { Bronchitis, tooth ache, insect bites, } \\
\text { dysentery,, hydrophobia }\end{array}$ \\
\hline 3 & Adhatoda vasica L. & Acanthaceae & Leaf & Bronchitis, diarrhoea, skin disease \\
\hline 4 & Alternanthera triandra Lam & Amaranthaceae & Stem, leaves & Lactagogue, washing the eyes \\
\hline 5 & Aloe barbadensis Mill. & Liliaceae & Leaves & Reduce constipation, skin disease \\
\hline 6 & Amaranthus spinosus Linn & Amaranthaceae & Leaves, stem & Gonorrhoea, monorrhoea, colic and eczama \\
\hline 7 & Amaranthus viridis Linn & Amaranthaceae & Entire plant, Roots & Fever, Intestinal gas, to cure constipation \\
\hline 8 & Argyreia nervosa Boj & Convolvulaceae & leaves & Healig Wounds, boils \\
\hline 9 & Argemone maxicana L & Papaveraceae & Yellow latex & $\begin{array}{c}\text { Leprosy, ulcers, jaundice, reduce } \\
\text { leucorrhoea }\end{array}$ \\
\hline 10 & Asparagus racemosus Willd & Liliaceae & Roots & $\begin{array}{c}\text { Gastric ulcers, indigestion, cooling agent, } \\
\text { diuretic, cough }\end{array}$ \\
\hline 11 & Bacopa monnieri Linn & Scrophulariaceae & Entire plant & Leucoderma, diuretic, bronchitis \\
\hline 12 & $\begin{array}{l}\text { Barleria cuspidata } \\
\text { B.Heyne ex Nees }\end{array}$ & Acanthaceae & Leaves & Antiseptic, tonic, joint pains \\
\hline 13 & Boerhaavia diffusa Linn & Nyctginaceae & Leaves, stem & $\begin{array}{l}\text { General health, anaemia, swellings cardiac } \\
\text { disorders, muscular pains, blood purifier }\end{array}$ \\
\hline 14 & Bryophyllum pinnatum Lam & Crassulaceae & Leaves & $\begin{array}{c}\text { Diarrhoea, dysentery, antiseptic, } \\
\text { Fever, cancer, headache }\end{array}$ \\
\hline 15 & $\begin{array}{l}\text { Cardiospermum helicacabum } \\
\text { Linn }\end{array}$ & Sapindaceae & Seeds, leaves, roots & $\begin{array}{l}\text { Arthritis, fever, neuropathy, } \\
\text { rheumatism, earache, eyesore }\end{array}$ \\
\hline 16 & Canna indica L. & Cannaceae & Leaves & Malaria, diarrhoea, fever \\
\hline 17 & Cassia auriculata Linn & Caesaplinaceae & Bark, leaves, fruits & Dysentery, asthma ,skin diseases \\
\hline 18 & Cassia tora Linn & Caesaplinaceae & Leaves and roots & $\begin{array}{l}\text { Dermatitis, ulcer, cough, fever, intestinal } \\
\text { problems, skin diseases }\end{array}$ \\
\hline 19 & Celosia argentea Linn & Amaranthaceae & Seeds & Diarrhoea \\
\hline 20 & Centella asiatica L & Umbelliferae & Stem and leaves & $\begin{array}{c}\text { Nervine tonic, spasmolytic skin disease, } \\
\text { leprosy, symphilis }\end{array}$ \\
\hline 21 & Citrus medica L. & Rutaceae & Fruit,shoots & Asthama, headache, stomach-ache \\
\hline 22 & Cleome viscose $\mathrm{L}$ & Capparidaceae & Seeds & Body pains \\
\hline 23 & Clitoria terntea L & Fabaceae & seeds & Laxative \\
\hline 24 & Commelina benghalensis L & Commelinaceae & Entire plant & Burns, boils, swellings, leprosy \\
\hline$\overline{25}$ & Croton tiglium L & Euphorbiaceae & seeds & $\begin{array}{c}\text { Cold, fever, cough, asthma, constipation, } \\
\text { dropsy }\end{array}$ \\
\hline 26 & $\begin{array}{l}\text { Commiphora mukul (Hook.Ex } \\
\text { stocks) }\end{array}$ & Burseraceae & Bark, leaves & Weight loss, antiseptic \\
\hline 27 & Cullen corylifolia $\mathrm{L}$ & Fabaceae & Seeds & $\begin{array}{c}\text { Leucoderma, leprosy, psoriasis, skin } \\
\text { diseases, hair treatment, diuretic, laxative. }\end{array}$ \\
\hline 28 & Cynadon dactylon $\mathrm{L}$ & Poaceae & Leaves and stem & Monorrhoea, Wound \\
\hline 29 & Cyperus rotundus $\mathrm{L}$ & Cyperaceae & Rhizome & Cough, Malarial fever, epilepsy, diarrhoea \\
\hline 30 & Datura metel L & Solanaceae & Leaves & Cough, fever, skin diseases \\
\hline 31 & Eclipa alba(1.) Hassak & Asteraceae & Leaves, stem & Ulcer and wounds in animals \\
\hline 32 & Euphorbia hirta Linn & Euphorbiaceae & Entire plant & Piles, gonorrhoea, cough, asthma \\
\hline 33 & Evolvulus alsionides & Convolvulaceae & Entire plant & Nervous tonic \\
\hline 34 & Gymnema sylvestris R.Br. & Apocynaceae & Leaves & Diabetics, weight loss \\
\hline 35 & Jatropha curcas Linn & Euphorbiaceae & Leaves and seeds & Rheumatism, scabies, ulcer, skin diseases \\
\hline 36 & Lantana camera var. aculeata & Verbenaceae & Stem and leaves & Cuts and wounds \\
\hline 37 & Lawsonia inermis L. & Lythraceae & Leaves & Diarrhoea, skin disease \\
\hline 38 & Martynia annua L & Martyniaceae & Leaves & Epilepsy \\
\hline 39 & Mentha spicata L. & Lamiaceae & Leaves & Headache, digestive disorders, fever \\
\hline 40 & Mentha viridis L & Lamiaceae & Leaves & Cooling agent, condiments \\
\hline 41 & Mimosa pudica L. & Fabaceae & Leaves & Antiseptic, diabetes \\
\hline 42 & Nerium oleander L. & Apocynaceae & Leaves, flowers & Treating ulcer, treat ringworm \\
\hline
\end{tabular}


ISSN No:-2456-2165

\begin{tabular}{|c|c|c|c|c|}
\hline 43 & Ocimum basilicum L & Lamiaceae & $\begin{array}{l}\text { Leaves, stem, } \\
\text { seeds }\end{array}$ & $\begin{array}{l}\text { Cooling agents, chronic dysentery, } \\
\text { diarrhoea, gonorrhoea }\end{array}$ \\
\hline 44 & Ocimum sanctum Linn & Lamiaceae & Leaves, stem & Catarrh and bronchitis, gastric disorders \\
\hline 45 & $\overline{\text { Phyllanthus niruri Linn }}$ & Euphorbiaceae & Entire plant & Diabetes, urinary infections, jaundice \\
\hline 46 & Plumbago zeylanica L. & Plumbaginaceae & Root & Skin problem, anti-cancer \\
\hline 47 & Portulaca oleracea L & Portulacaceae & Entire plant & Diarrhoea, diabetes, headache, dysentry \\
\hline 48 & $\underline{\text { Ricinus communis Linn }}$ & Euphorbiceae & Leaves and seeds & $\begin{array}{c}\text { Laxative, rheumatism, skin diseases, ulcers, } \\
\text { boils, headache }\end{array}$ \\
\hline 49 & Sida cordifolia (Burm. F) Borss & Malvaceae & Entire plant & Gonorrhoea, leucorrhoea \\
\hline 50 & Spilanthes acemella L & Asteraceae & Flower & Treating toothache \\
\hline 51 & Tephrosia purpurea Pers & Fabaceae & Entire plant & $\begin{array}{l}\text { Asthma, piles Gonorrhoea, bronchitis, } \\
\text { anaemia,toothache }\end{array}$ \\
\hline 52 & $\begin{array}{c}\text { Tinospora cordiflora Willd.Var. } \\
\text { javanica (k unth.) Baker in J. } \\
\text { Linn } \\
\text { Soc. Bot }\end{array}$ & Menispermaceae & Leaves, Bark & $\begin{array}{l}\text { Fever, urinary diseases, diarrhoea, } \\
\text { dysentery, piles, gonorrhoea }\end{array}$ \\
\hline 53 & Tribulus terrestris L & Zygophyllaceae & Entire plant & Dysuria, stomach ache, Urinary disorders \\
\hline 54 & Tridax procumbens L. & Asteraceae & Leaves & Maleria, diarrhoea boils, wounds, cooling \\
\hline 55 & Triumfetta rhomboidea Jac & Tiliaceae & $\begin{array}{l}\text { Leaves, flowers and } \\
\text { fruits }\end{array}$ & Gonorrhoea \\
\hline 56 & Vitex negundo Linn & Verbenaceae & Leaves, & Rheumatism \\
\hline 57 & Withania sominifera L. & Solanaceae & Roots & $\begin{array}{l}\text { Anti-Cancer, Reduce Cholesterol level, } \\
\text { stress }\end{array}$ \\
\hline 58 & Xanthium strumarium L & Asteraceae & Leaves and fruits & Tuberculosis, small pox, laxative \\
\hline
\end{tabular}

\section{CONCLUSION}

In the folklore system of herbal drugs, various herbs are used for therapeutic purposes. Present studies revealed that, this study area is irrigated throughout the year and so reach in biodiversity of medicinal plants. During present investigations, a total 58 species belonging to 35 families were encountered. Various plant parts of these plants are used by the people as crude drugs commonly. Present investigation will be benefited to the people those who are interested in folkloreherbal medicine.

\section{REFERENCES}

[1]. Auti, B.K. 2011. Medicinal flora of Angiosperms from Jeur (Bayajabai), District Ahmednagar (M.S.). Advances in Plant Sciences, 24 ( I ):347-349.

[2]. Basu and Ramsankar. 2007. Folklore values of weeds grown in wastelands of Vedharanyam and Kodiakarai, Nagapattinam district of TamilNadu. Advances in Plant Sciences, 20(II):251-254.

[3]. Behera, L. M. and Sen, S. K. 2007. Traditional use of some plants against Gynaecological disorders by the tribals of Ramkhol village forest of barapahad hill range in bargarh District (Orissa). Advances in Plant Sciences, 20(II): 255-257.

[4]. Cook, T. 1903. The Flora of Presidency of Bombay. Vol. I, II \&III. BSI, Calcutta.

[5]. Deokar, A.B. 1998. Medicinal Plants. D.S. Mana Vikas Foundation, Pune.
[6]. Kirtikar, K.R. and Basu, B.D. 1984. Indian Medicinal plants Vol. I to III. Bishen Singh Mahendra Pal Singh, 23-A, New Connaught Place Dehradun.

[7]. Kokate, C. K., Purohit A. P. and Gokhale, S.B. 2016. Pharmacognosy. Nirali Prakshan, Pune. pp.1-910.

[8]. Mohapatra, H. K. and Behra, L. M. 2011. Medicinal Plants Along the water stream of Pradhanpat water fall of Deogarh forest range in Deogarh district (Orissa). Advances in Plant Sciences, 24(I): 251-256.

[9]. Pradhan, S.G. and Singh, N.P. 1999. Flora of Ahmednagar District (Maharashtra). Bishen Singh Mahendra Pal Singh,23-A, New Connaught Place, Dehradun.

[10]. Rajaram, N., Prakash, R. and Sharmila, S. 2014. Herbal remedies for common Ailments prevailing in rural areas of Palladam and Perumanallum,Tiruppur district,Tamil Nadu. Advances in Plant Sciences, 27(I):165-169.

[11]. Rao, J.R.,.Jena, P.K and H.P.Sahoo. 2011. Indigenous Phytotherapy for Gastro-Intestinal Disorders among tribals of Dhenkanal district. Orissa. Advances in Plant Sciences, 24(I):361-364.

[12]. Sutaria, A.N. 1962. A text book of Systematic Botany, Khadayata Book Depot, Ahmedabad. pp. 1-626.

[13]. Tripathi, A. K., Kumar, V., Tiwari, R.K.S. and Tiwari, R. B. 2007. Medicinal plants of Northern Hill Region of Chhattisgarh and their uses. Advances in Plant Sciences, 20(I): 221-226. 